\title{
PARALELISMOS, IDENTIDAD Y ALTERIDAD EN LA VITA SPYRIDONIS ATRIBUIDA A LEONCIO DE NEÁPOLIS
}

\author{
ANALÍA V. SAPERE \\ UBA-CONICET. Argentina
}

Resumen: El artículo se propone un análisis de la Vida de Espiridón atribuida a Leoncio de Neápolis (s. VII d. C.). Se estudian especialmente los paralelimos que se establecen entre Espiridón y los personajes episódicos, para determinar de qué modo influyen en la caracterización del santo y, en última instancia, comprobar su valor didáctico en la obra.

Palabras clave: Vita Spyridonis, Leoncio de Neápolis, hagiografía, paralelismo, didactismo.

\section{PARALLELISMS, IDENTITY AND ALTERITY IN THE VITA SPYRIDONIS ATTRIBUTED TO LEONTIUS OF NEAPOLIS}

\begin{abstract}
This paper aims to analyze the Life of Spyridon attributed to Leontius of Neapolis (7th century AD). We will study in particular parallelisms between Saint Spyridon and the episodic characters in order to define how this technique endeavors to outline the description of the saint and to verify its didactic value in the text.
\end{abstract}

Keywords: Vita Spyridonis, Leontius of Neapolis, hagiography, parallelism, didactism

Recibido:1.03.2016 - Aceptado: 28.03.2016

\section{Correspondencia: Analía V. Sapere}

Email: analiasapere@gmail.com

Doctorado en Letras. Universidad de Buenos Aires con orientación en Letras clásicas. Profesora de griego en la misma Universidad. Profesora de Griego y Latín 1 en la Universidad del Salvador. Becaria postdoctoral del Consejo Nacional de Investigaciones

Científicas y Técnicas (Conicet). 
Introducción ${ }^{1}$

T a Vida de Espiridón atribuida a Leoncio de Neápolis (s. VII a. C. $)^{2}$ se enmarca dentro de los cánones tradicionales de la hagiografía, dado que narra en un lenguaje comprensible a todo público los hechos correspondientes a la vida del obispo de Chipre (270-348) con un firme propósito edificante. A su vez, se plasman en la biografía una serie de tópicos, tales como la humildad del santo, su desprecio por los bienes materiales, la realización de milagros y la ayuda a los necesitados, erigiéndose como modelo de conducta y custodio de los valores de la cristiandad ${ }^{3}$. Todos estos tópicos aparecen desplegados a través de las narraciones episódicas, que son el núcleo de la obra, y de las que nos ocuparemos aquí. En efecto, en el presente trabajo proponemos un análisis de los distintos episodios que conforman la biografía de Espiridón, a fin de indagar de forma particular en uno de los mecanismos discursivos allí planteados: el paralelismo. Para ello, rastrearemos y analizaremos las interacciones del santo con los diferentes personajes episódicos, con la intención de entender de qué modo estas contribuyen a describir, ya por oposición ya por identificación, la figura del santo. Entendemos que el recorrido narrativo conformado en dichas interacciones no es azaroso, sino que intenta reflejar rasgos del santo que afloran a partir del paralelismo. En algunos casos, la comparación implica afinidad; en otros, contraste y alteridad. Nuestro desafío consistirá en interpretar tales relaciones en el contexto didáctico-moralizante de la obra. Es importante destacar que, dada la brevedad de la biografía, el trabajo se hará sobre la totalidad de los episodios, lo que nos permitirá extraer conclusiones con gran sustento textual ${ }^{4}$.

1 Una primera versión de este trabajo fue leída en las Primeras Jornadas Nacionales e Internacionales de Ficcionalización y Narración en la Antigüedad, el Tardoantiguo y el Medioevo "Un milenio de contar historias" (Buenos Aires).

2 Hay controversia respecto de la autoría. Van den Ven (1953), por ejemplo, no reconoce que Leoncio sea su autor y la edita bajo el nombre de "Vida II"; en cambio Garitte (1955), con quien coincidimos, afirma que sí fue escrita por Leoncio de Neápolis. Cf. también Longo $(2004,218)$. Nuestra postura encuentra su fundamento en las investigaciones realizadas en el marco del Proyecto UBACyT 01W106. Cf. Cavallero $(2014,15-18)$.

3 Acerca de estos elementos tópicos del género hagiográfico, cf. Pratsch (2005), Bourbouhakis-Nilsson (2010), Hägg (2011 y 2012), entre otros.

4 Utilizaremos el texto y la numeración de la edición de van den Ven (1953). Las traducciones son de Cavallero et al. (2014). 


\section{Acerca del paralelismo}

La técnica del paralelismo aplicada al género biográfico encuentra su mayor representante en Plutarco de Queronea, quien dedica su obra Bío П $\alpha \rho \alpha ́ \lambda \lambda \eta \lambda$ o a narrar la vida de personajes griegos y romanos para luego contraponerlos y, a partir de la comparación o sýnkrisis, extraer elementos que sirvan de enseñanza a sus lectores ${ }^{5}$. Desde luego que no es Plutarco quien inventa dicha técnica; ésta se remonta a los ejercicios de retórica conocidos como $\pi \rho \gamma \cup \mu v \alpha ́ \sigma \mu \alpha \tau \alpha$ y a los tratados de retórica clásicos. Encontramos también ejemplos de ella en Isócrates, Polibio, Varrón y Nepote, quienes hacen uso del paralelismo o comparación para conferir a sus textos un atractivo retórico y a la vez dirigir sus palabras en favor o en contra del personaje retratado. La riqueza del procedimiento consiste, pues, en explotar todo tipo de comparaciones y encauzarlas hacia un fin determinado, que varía de acuerdo con la obra. El procedimiento más común es el de poner en paralelo dos personajes similares para enaltecer a uno de ellos o lo contrario, es decir, establecer paralelismos por contraposición con la pretensión de ensalzar a uno y desprestigiar a otro (Kennedy, 2003, 162 ss.). Dependerá luego de la maestría literaria del autor lograr que en dicho esquema prefijado se presenten elementos originales para plasmar una obra de interés del auditorio, y no caer en lugares comunes altamente conocidos. Los autores del Nuevo Testamento también han sabido dar uso a la sýnkrisis (Shipp, 2005, 153; Neyrey, 2009 y n. 62; Gorman, 2015, 56 ss.), de modo que no ha de extrañarnos su empleo en la tradición cristiana posterior. En el caso particular de la literatura bizantina, no es necesario ahondar en la importancia que los autores conferían al estudio de la retórica, y allí los $\pi \rho о \gamma v \mu v \alpha ́ \sigma \mu \alpha \tau \alpha$ ejercieron una gran influencia, sobre todo a partir de las obras de Hermógenes, Teon, Aftonio y Libanio ${ }^{6}$.

En el caso de la Vida de Espirdón, el paralelismo se establece entre el personaje central del texto y los personajes episódicos con los que el santo se va encontrando a lo largo de la narración. Se advierte, en primer lugar, un trabajo en relación con la estructura narrativa, dado que el autor parece concentrar los paralelismos por identidad en la primera parte de la hagiografía, mientras que hacia el final nos muestra los paralelismos por oposición. Veremos a continuación cómo se desarrollan ambos procedimientos textuales.

$5 \quad$ Cf. Erbse (1956), Pelling (1986), Larmour (1992), Swain (1992), Harrison (1995), Duff (2000), Beck (2002).

6 Sobre la influencia de la retórica clásica y de los progymnasmata en la literatura bizantina, cf. por ejemplo Hunger (1978), Kennedy (1999, 186 ss.), Kustas (2001), Kennedy (2003, xii), Jeffreys (2003), Whitby (2010). 
ANALÍA V. SAPERE: Paralelismos, identidad y alteridad en la Vita Spyridonis...

2. Paralelismos por identificación: la importancia didáctica de la afinidad.

Decíamos entonces que a lo largo de los primeros capítulos de la hagiografía observaremos que se plantea una identificación explícita entre el santo y los personajes con los que interactúa (capítulos 1 a 9). Pasemos a analizar caso por caso en qué consiste tal identificación, así como su funcionalidad estratégica en la obra.

El primero de los milagros narrados tiene lugar en el seno familiar, pues interviene Irene, la hija fallecida del santo. El milagro no reviste mayor complejidad que la de la aparición de la muchacha, después de muerta, para indicarle a su padre dónde se hallaba un objeto que era necesario devolver a su dueña. El autor se encarga de asimilar las características comunes de ambos personajes, que pueden resumirse en la pureza de padre e hija: en cuatro oportunidades se menciona la virginidad de la muchacha ( $\pi \alpha \rho \theta \varepsilon \dot{v}$ os, en 105:

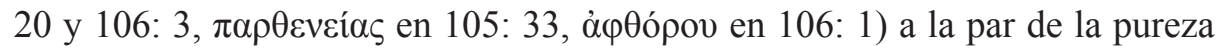

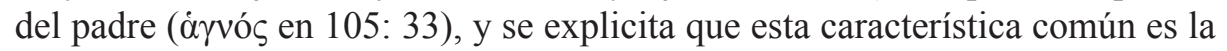

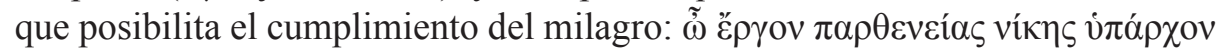
$\sigma \hat{\jmath} \mu \beta o \lambda o v^{7}$. Dado que es un capítulo introductorio, porque se narra allí el primer milagro, no podemos dejar de pensar en lo significativo que resulta que sea esta virtud del santo la que se desea poner en evidencia.

Los capítulos que siguen a continuación (2, 3, 4 y 6) también nos presentan personajes que entablan una relación de identificación con Espiridón, pues comparten con él la pertenencia al mundo del campo ${ }^{8}$. Los ejemplos más significativos se hallan en los capítulos 3 y 4, cuando dos pobres campesinos requieren su ayuda a causa del perjuicio ocasionado por el personaje del rico avaro. Por un lado, entonces, la identificación se basa en la actividad en común de estos y Espiridón, pues sabemos gracias a las palabras introductorias del

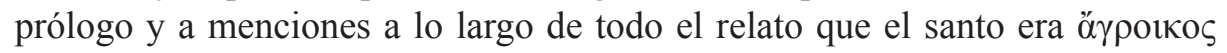
(campesino), al igual que ellos. Esta identificación es tácita, es decir, no aparece marcada en el texto: el lector debe establecer la conexión entre las referencias a la actividad atribuida a Espiridón y la actividad de estos personajes - lo que de todas formas resulta muy sencillo, por la profusión de dichas menciones: 104:

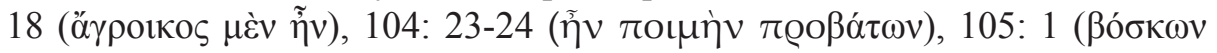

$7 \quad$ Dice Kazhdan (1990: 132): “On the scale of Byzantine ethical values, virginity occupied the topmost place, much higher than legitimate marriage". Más adelante, agrega: "Hagiography presented a way of compromise between filial obedience and pious chastity".

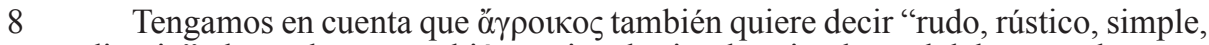
ordinario", de modo que también sugiere la simpleza intelectual del santo, elemento tópico del género. Cf. Rubenson (2000). 


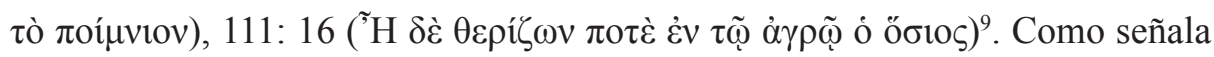
Rousseau (1999, 52), el hecho de introducir en las hagiografías personajes y situaciones mundanos es un ejemplo de la intencionalidad retórica de construir a un personaje mucho más cercano y accesible al público en general, lo que aquí se pone en evidencia por tratarse de los capítulos iniciales, en los que el público comienza a conocer al personaje $\mathrm{e}^{10}$.

Existe además una identificación explícita con estos personajes, enunciada en boca del propio Espiridón, cuando empatiza con el campesino del capítulo 4 por la pobreza que aqueja a ambos y le dice (109: 4): "No te lamentes, hijo, teniendo fe en Dios, pues también yo siempre convivo con la

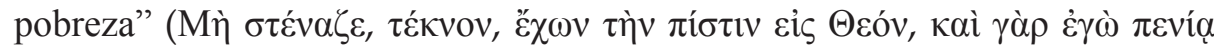

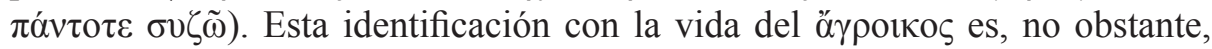
matizada desde un comienzo, porque Espiridón no es un simple campesino: en el texto queda establecido además un paralelismo entre la actividad de pastoreo literal y la metafórica, imagen que es retomada a lo largo de todo el relato ("apacentando en la noche el rebaño y pastoreando en el día a los hombres", "a unos y a otros les proveía sus alimentos, tanto a las irracionales criaturas, cuanto a las racionales ovejas de la Iglesia") ${ }^{11}$. En suma, Espiridón se identifica con los pobres campesinos con quienes comparte una forma de vida, pero el autor enuncia claramente la peculiaridad de la condición del santo, que no puede ser reducida a la de un ốyporkos como los demás.

Respecto de esta identificación con los hombres de campo podemos mencionar también el capítulo 2. Lo separamos de los ejemplos anteriores porque no aparece en él un personaje principal con el que Espiridón entable relación. Así, pues, en el capítulo 2 no hay mención de ningún personaje

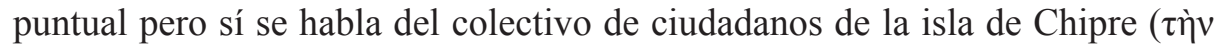

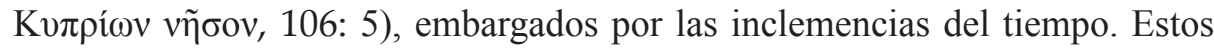
aparecen mencionados como "la multitud" ( $\tau$ oĩ $\varsigma$ o $\lambda \lambda$ oĩ $\varsigma$ ), los "esclavos que

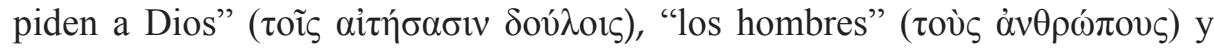

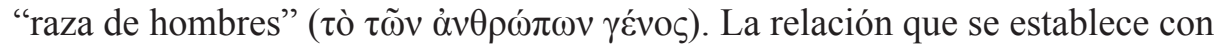
los demás episodios analizados es que el milagro llevado a cabo por Espiridón

$9 \quad$ Cf. también los capítulos 10 y 17, donde se narra acerca de sus rebaños y cómo se ocupaba de ellos.

10 Acerca de la identificación autor-auditorio cf. también Sapere-Villahoz (2014).

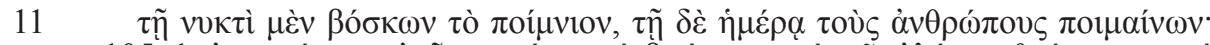

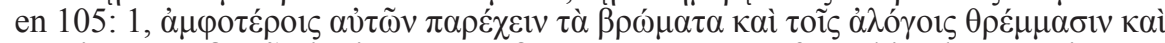

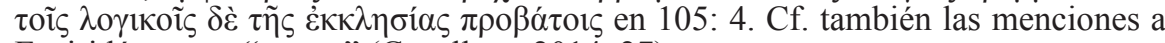
Espiridón como "pastor" (Cavallero, 2014, 27). 
es el pedido de agua ante la sequía y luego el pedido de cese de las abundantes lluvias, lo que conecta al personaje con la vida de campo. En efecto, allí queda clara la súplica de Espiridón a Dios para que la tierra dé frutos (îv $\alpha \tau$ ñn

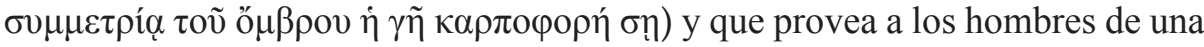

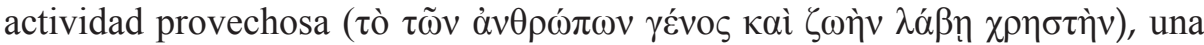
clara alusión a la agricultura, elemento central de estos primeros capítulos.

Lo mismo podemos decir del capítulo 6, que tiene lugar en un escenario agreste, con un grupo de campesinos: Espiridón se encontraba cosechando en el

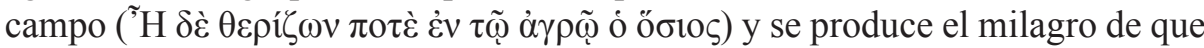
finas gotas de agua caen sólo sobre él, mostrando cambios de color en el cabello. Como ocurre en el capítulo 2, aquí no hallamos paralelismo con un personaje concreto, sino que el santo se identifica con el grupo de hombres con los que realiza la actividad de la cosecha, mencionados como "los otros presentes"

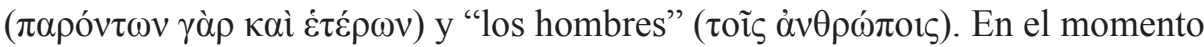
de interpretar el curioso milagro, Espiridón se dirige a ellos como "hermanos"

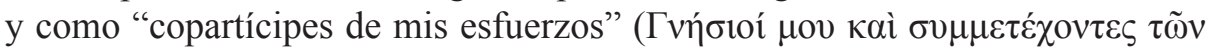

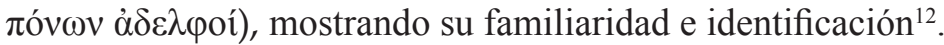

Hay otros dos episodios que, a nuestro entender, comportan también una identificación entre Espiridón y los personajes que allí aparecen, aunque de distinta índole respecto de los analizados. Se trata del paralelismo que se establece con dos figuras de poder: el gobernador de Constanza en el capítulo 5 y el emperador en el capítulo $8^{13}$. En el caso del capítulo 5, Espiridón acude a la ciudad de Constanza para auxiliar a un amigo que estaba preso injustamente.

12 "Brother and sister appellations for men and women who are not sibilings have a long tradition in the ancient world, in social and religious contexts. Their meaning may be stable, analogous to a title or honorific, or fluid, depending on context. In direct address, they express notional equiality or a claim to affective familiarity, regardless of their kinhio relation. [...] The erasure of social hierarchies in early Christianity had its root in the twofold belief that all Christian men and women shared a common descent form God the Father, ant that they shared brotherhood with Christ throogh the Incarnation" (Rapp, 2016, 6-7).

13 Esta identificación no debe llamarnos la atención. Como dice Patlagean (1968, 108-9): "Certes, les pauvres et les simples jouent un rôle important dans les Vies et Miracles, à l'image de la société réelle, et selon la pente de la pensée chrétienne. Mais les auteurs insistent sur la diversité sociale des personnes qui seront en rapport avec le saint, paysans des terres du monastère, ou des environs, citadins des villes voisines, de toutes conditions, personnages de la cour et hauts fonctionnaires, quand ce n'est pas l'empereur lui-même. Les hagiographes veulent s'adresser à toutes les classes sociales, parce que leur but est d'inculquer à la société entière la vénération du groupe monacal, et, localement, du monastère, ou du sanctuaire; et en effet, par un biais ou par un autre, c'est la société entière qui a affaire à eux". 
La resolución de este segmento narrativo es curiosa, dado que Espiridón no hace ninguna acción concreta respecto del conflicto, salvo la mostración de su poder: cuando se dirige hacia Constanza, donde se encontraba el reo, la crecida de un río ${ }^{14}$ les impide avanzar a él y a los suyos, por lo que obra el milagro de la contención de las aguas. Espiridón no llega a Constanza aún y ya es célebre la concreción del milagro (transmitido por los testigos), que es lo que persuade al gobernador del lugar para liberar al amigo. El poder de la gracia del santo

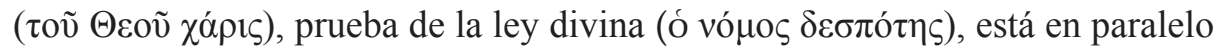
con el poder terrenal del gobernador, quien decide sobre la justicia humana (ó $\alpha v \theta \rho \omega ́ \pi v v \varsigma$ vó $\mu$ os); de hecho, con este paralelismo explícito se cierra el capítulo $^{15}$. La identificación de dos planos de poder resulta en una superioridad por parte de Espiridón, pues consigue, como intercesor de la ley divina, su propósito de liberar al encerrado injustamente, cuando la ley humana estaba equivocada, porque había juzgado culpable a un inocente.

En el capítulo 8 Espiridón se encuentra con el emperador ${ }^{16}$, quien lo había llamado para que sanara el padecimiento que lo aquejaba. La identificación queda en evidencia sobre todo por el paralelismo de las descripciones de ambos. Por un lado, el autor se encarga de magnificar la figura del emperador: en uno de los pocos capítulos en los que presenciamos descripciones extensas ${ }^{17}$, se nos habla en detalle de la corte del rey, del lujo y de su imponente y destacada figura. Así, por ejemplo:

Teniendo en círculo muy jóvenes espadachines, ornados con oro, revestidos radiantemente en armas; y a otros ubicados a su vez temerosamente, con rostro entristecido y cabeza inclinada hacia abajo; y otros varones, sirvientes delgados como cabellos, con túnicas doradas y adornados con calzado del estilo; y otros portadores de cayados y

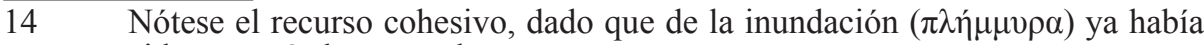
aparecido en capítulos precedentes.

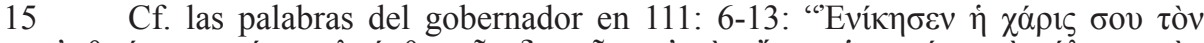

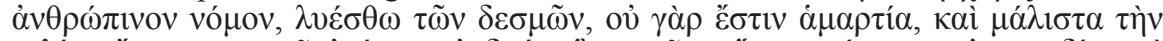

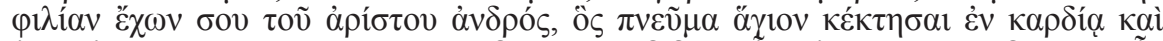

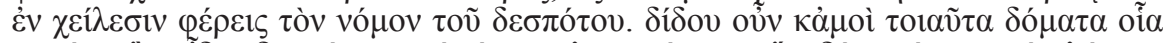

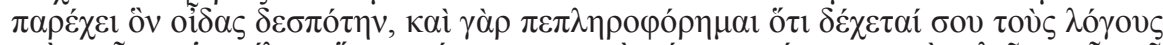

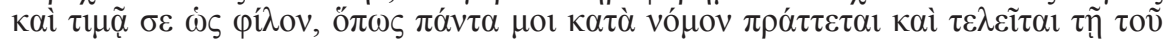
$\Theta \varepsilon \circ \chi^{2} \chi \dot{\alpha} \rho \imath \tau \imath "$.

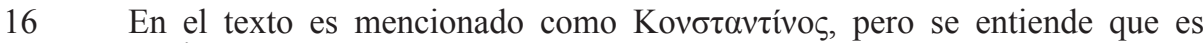
Constancio II.

17 Tengamos en cuenta que, dado el tipo de narración, breve, simple y concisa, el autor no suele detenerse en descripciones elaboradas. 
toda por así decir dignidad de pequeños y grandes, presentes, sin voz y con mucho miedo $(114: 1-8)^{18}$.

Refulgía por la púrpura y brillaba con piedras como sol, sentado sobre un sublime estrado, suficiente para pasmar la mente de los hombres por su visión $(113: 30-114: 1)^{19}$.

Por otro lado, Espiridón es descripto como una contrapartida de éste: también tiene un séquito, conformado por su discípulo Trifilio ("Teniendo

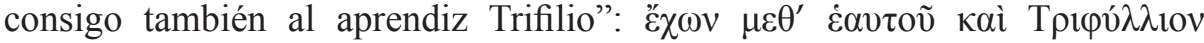

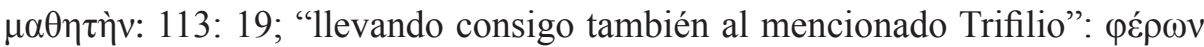

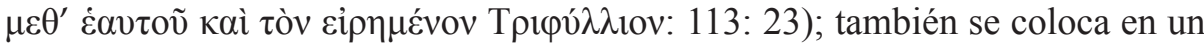
lugar preponderante ("Se manifestaba a los grandes varones como brillante y

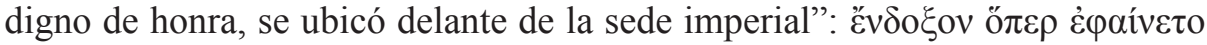

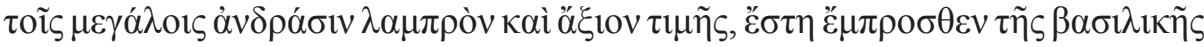
$\kappa \alpha \theta \varepsilon ́ \delta \rho \alpha \varsigma: 113: 27-9)$, y también se describe en detalle su figura (lo que ocurre solamente aquí):

El emperador, cuando vio al piadoso, enseguida reconoció al que antes buscaba, que llevaba en manos un bastón de pastor y en la cabeza una capucha acabada en frutos de palmas, como en una canasta, y a la vez, otra cosa, como un frasquito angosto colmado de aceite $(114: 10 \mathrm{ss})^{20}$.

De este modo queda subrayada la distinción de Espiridón, en tanto que su acción sanadora es efectiva, a diferencia de la de los demás convocados, que resultaron un fracaso $^{21}$. Nuevamente se enfrentan dos planos de poder:

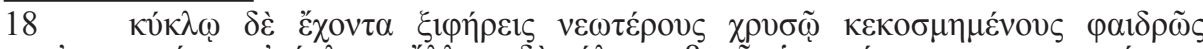

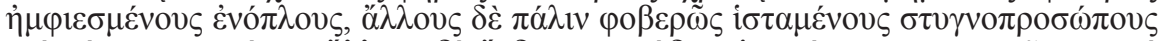

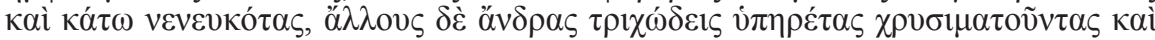

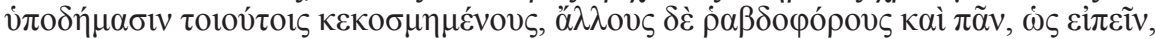

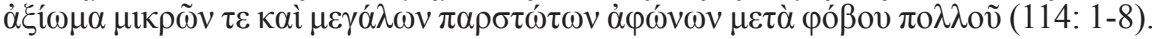

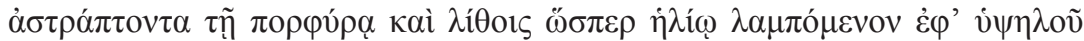

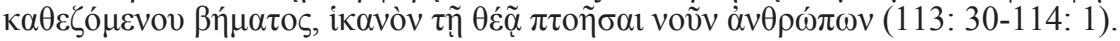

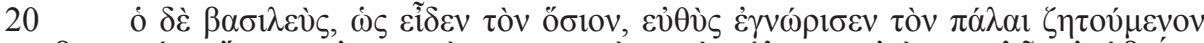

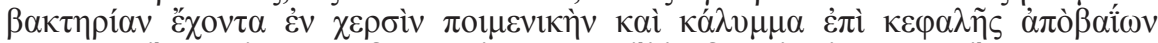

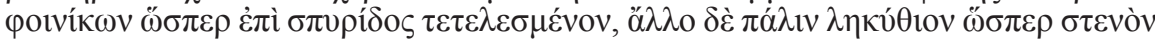

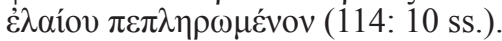

21 Hay todo un trabajo discursivo que tiende a comparar a Espiridón con los demás médicos que trataron al emperador; estos eran excelentes y virtuosos, por

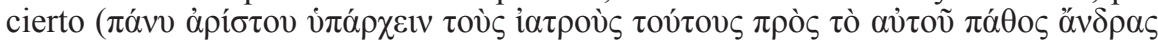


el poderío terrenal, con el lujo y la ostentación, y el poderío divino, que es el único capaz de sanar, humilde y desinteresadamente. Y como en el episodio del gobernador, de la identificación se pasa a la manifestación de la superioridad del santo: las obras de generosidad que realiza Espiridón luego del milagro causan admiración en el emperador ( $\dot{\varepsilon} \theta \alpha v ́ \mu \alpha \zeta \varepsilon v)$ y representan para él una enseñanza

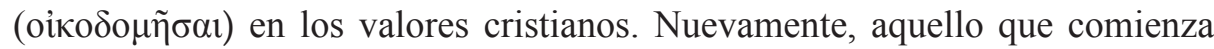
como una identificación, termina revelando la abismal diferencia entre el poder ejercido por la autoridad terrenal y el poder del que es amigo e instrumento de $\operatorname{Dios}^{22}$.

Un último ejemplo de identificación aparece en el capítulo 9, esta vez, con la figura de Artemidoro. Una mujer se acerca a Espiridón para pedir su ayuda, porque acaba de morir su hijo recién nacido. En dicha ocasión, Espiridón estaba acompañado por Artemidoro, de quien se dice que "servía a Cristo con sus luchas, con muy virtuosa conducta y deseaba pasar inadvertido

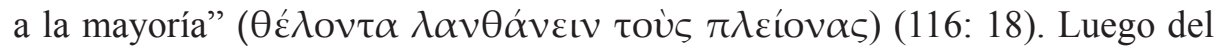
milagro de la resucitación, Espiridón ruega a Dios que el hecho no sea conocido

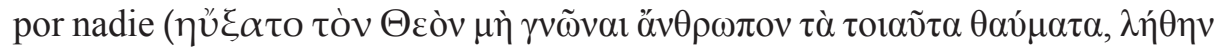

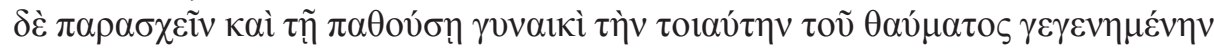
$\chi \alpha ́ \rho ı v)$, con lo que iguala su comportamiento al de Artemidoro, quien, como dijimos deseaba mantenerse oculto. La decisión de actuar en secreto llama la atención de cualquier lector, dado que ni antes ni después en la obra se menciona en la hagiografía esta pretensión del santo de no dar a conocer sus obras benéficas y milagros, sino que, de hecho, ocurre más bien todo lo contrario, pues la exhibición de los milagros forma parte esencial de su paideía. A modo de ejemplo, recordemos lo ocurrido en el capítulo 5 ya analizado, en el que es fundamental que se difunda la noticia del milagro acaecido para persuadir al gobernador; asimismo, hay numerosos pasajes de la biografía que prueban la importancia de la exhibición del obrar milagroso, dado que de esta forma se difunde el mensaje de santidad ${ }^{23}$ (que no es otra cosa que un reflejo de la

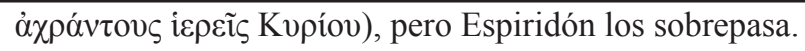

22 La violencia con la que se cierra el episodio pone en evidencia la diferenciación entre Espiridón y el emperador: "El justo sale corriendo de allí así desnudo, como huyendo de una fiera mortífera, hacia tierra extranjera o sea su propia patria, sacudiéndose de sí toda avaricia por el oro, para edificar incluso con el ejemplo al

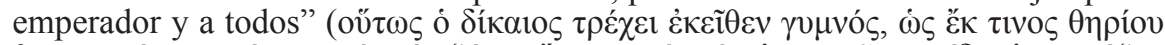

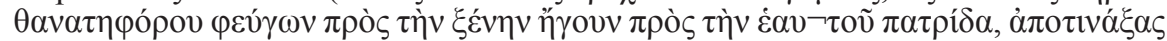

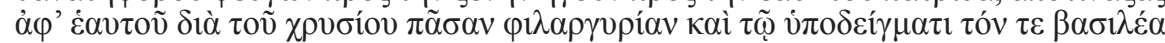

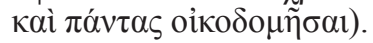

23 Cf. cap. $6,7,8.11,12,13$, en los cuales la publicidad de la acción del santo 
ANALÍA V. SAPERE: Paralelismos, identidad y alteridad en la Vita Spyridonis...

actividad del hagiógrafo).

\section{Paralelismos por oposición: acercamiento a la alteridad}

En los capítulos que siguen a continuación encontraremos, en cambio, una relación de alteridad entre Espiridón y los distintos personajes que aparecen. Los casos más evidentes son aquellos en los que el personaje debe efectuar una reprimenda o castigo frente a los pecadores, como el matarife que había llevado sin pagar una cabra del rebaño del santo (cap. 10), Trifilio, cuando se ve poseído por el deseo de bienes materiales (cap. 14), la adúltera (cap. 15), la prostituta (cap. 7) o los ladrones del establo (cap. 17). Pero a pesar de que la acción pecaminosa representa un claro contraejemplo de santidad, Espiridón se acerca a ellos a través de palabras y gestos amenos, les da el perdón y en algunos casos les muestra gestos de amistad.

En el capítulo 10, por ejemplo, se enfrenta a quien le roba una de sus cabras para advertirle acerca del error, pero no reprende al ladrón, sino que lo persuade para revisar su falta con un tono suave y comprensivo: "Hijo, no combatas, sino que, yendo a la casa, controla el pago de todas las cabras, no sea que, por olvido, te hayas ido sin dar el pago de ésta". En el caso del robo del

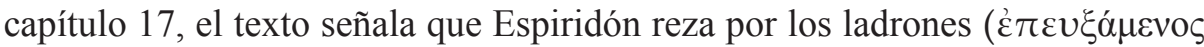

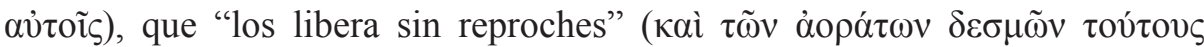

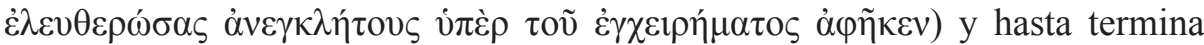
ofreciéndoles un cordero, "como recompensa de la fatiga" ( $\Lambda \alpha ́ \beta \varepsilon \tau \varepsilon, \tau \varepsilon ́ \kappa v \alpha, \tau o \tilde{v}$

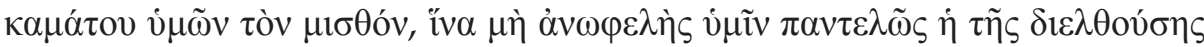

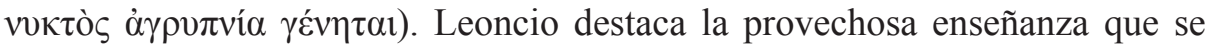
desprende de dicha actitud de Espiridón ( $\left.\dot{\varphi} \varphi \varepsilon \lambda \varepsilon^{\prime} \alpha \varsigma \pi 0 \lambda \lambda \tilde{\eta} \varsigma\right)$, en la medida en

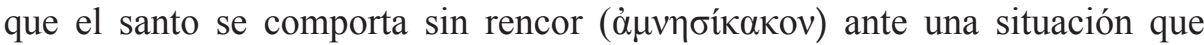
perfectamente hubiera ameritado un castigo de su parte. Asimismo, en el capítulo 14, Espiridón dirige cordiales palabras a Trifilio, luego de enterarse de manera milagrosa de su pecado de codicia, pues lo aleja de esos pensamientos recordándole los mandamientos de Dios, pero sin reprenderlo ${ }^{24}$.

Dentro de este grupo de episodios en los que Espiridón se encuentra con pecadores, hay dos que nos interesan particularmente: los capítulos 15 y

es necesaria para que resulte completamente efectiva, en tanto testimonio de la divinidad.

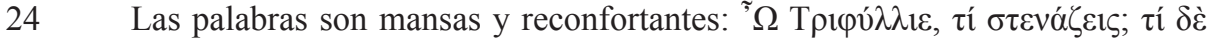

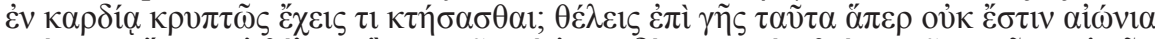

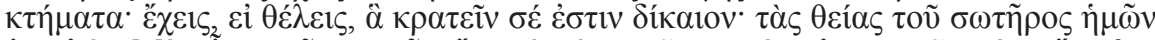

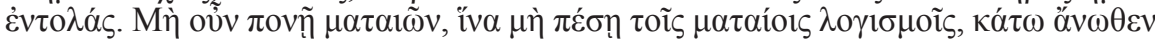

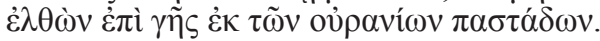


17. En el capítulo 15 se narra el episodio en el que es condenada una esposa adúltera: luego de conocer el acto pecaminoso, Espiridón le dirige muy duras palabras: "No acabarás, mujer, lo que adquiriste malamente, al arruinar el

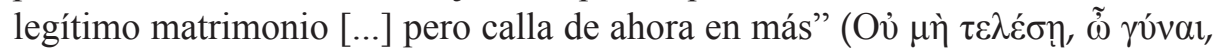

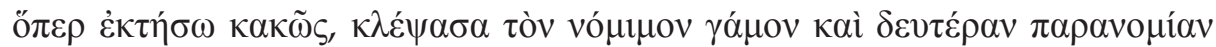

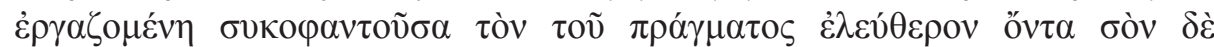

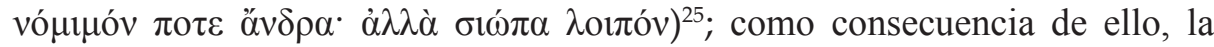

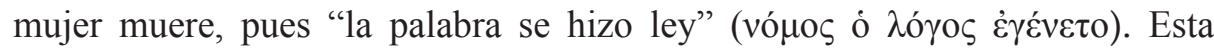

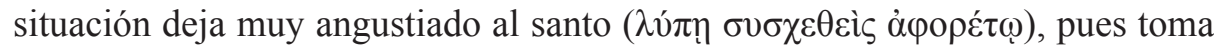
consciencia del poder performativo de sus dichos ( $\varepsilon_{\mu} \mu \alpha \varepsilon v$ ), lo que lo lleva a replantear su conducta a partir de ese momento. Reflexiona así el santo: "No juzgaré desde ahora, puesto que tengo la palabra de los labios como un puñal,

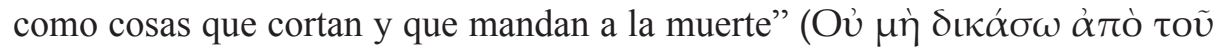

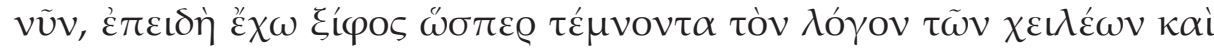
$\pi \alpha \varrho \alpha \pi \varepsilon \dot{\varepsilon} \mu \pi \mathrm{O} \tau \tau \alpha \tau \tilde{\omega} \theta \alpha \nu \alpha \dot{\tau} \omega)^{26}$. En el capítulo 7, una prostituta se acerca a Espiridón para lavar sus pies, pero éste la rechaza, porque reconoce su vida de

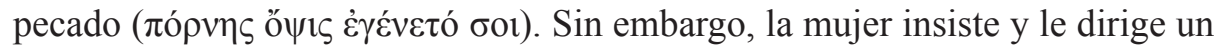
extenso parlamento, en el que reconoce su falta y pide perdón. Las palabras conmueven por completo al santo, quien inmediatamente cambia su actitud

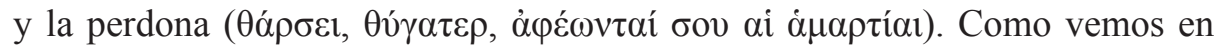
ambos casos, Espiridón, como consecuencia del encuentro, reflexiona sobre sus hechos al punto de arrepentirse o modificar su actitud. No son precisamente modelos de conducta los que llevan al santo a esta reflexión, de modo que una vez más vemos matizada la relación de alteridad: hasta de los peores pecadores es posible recibir una enseñanza.

$25 \quad$ Esta severa actitud es representativa de la época; como afirma Kazhdan (1990, 135): "In Byzantine society, which venerated marriage and was frightened by the sight of the naked body, adultery and lewdness occupied a surprisingly important place [...]. The secular administration took severe measures to fight this evil. If we may believe the Vita of Antony the Younger, a governor issued a decree ordering every fornicator and every prostitute to be arrested, their property confiscated, and their hair cut off to make them a laughing stock". Acerca de los antecedentes legales de las duras condenas a los adúlteros en Bizancio, cf. Youni (2001).

26 El paralelismo por oposición está construido desde la cuidada selección léxica

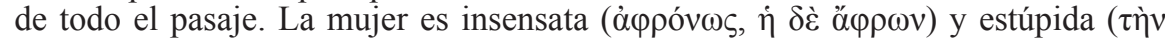

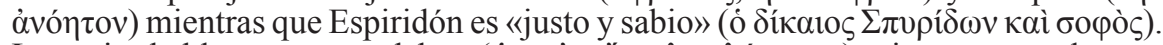

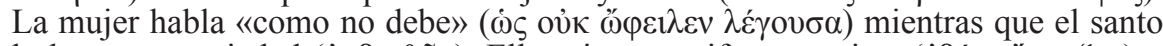

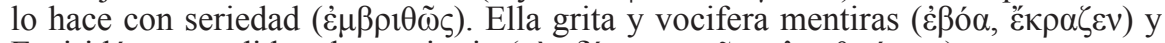

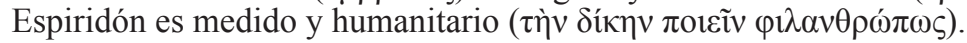


También representan una alteridad a la figura del santo los hombres congregados en la iglesia en el momento de las ceremonias religiosas narradas en los capítulos 11 y 12. Esta alteridad no responde, en rigor, al modelo justo/ pecadores que acabamos de analizar; en estos casos, los personajes aludidos significan una oposición y contraste respecto de Espiridón pues toman una actitud desafiante. En el capítulo 11, por ejemplo, se narra el episodio del diácono desobediente: Espiridón le había pedido que hiciera una oración breve (

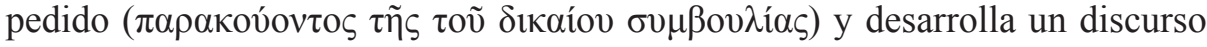
"en multitud de palabras" ( $\dot{\varepsilon} v \pi \lambda \eta \dot{\eta} \theta \varepsilon \imath \lambda \hat{\gamma} \gamma \omega v)$. Leoncio está interesado en destacar la falta del diácono, al repetir inmediatamente a continuación su falta de consideración hacia el pedido del santo: "La luminosidad del sol era muy gravosa y era doble quemazón la intención del que hablaba, desoyendo

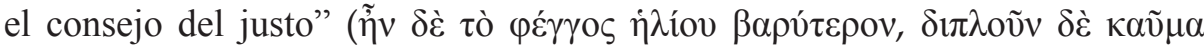

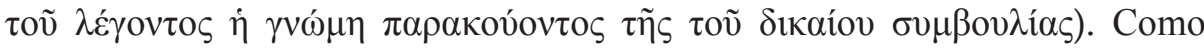

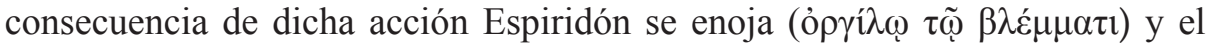
diácono recibe como castigo la pérdida completa de su voz (ó $\varphi \omega v o s)$. Un segundo ejemplo lo hallamos en el capítulo 12: en el comienzo se menciona

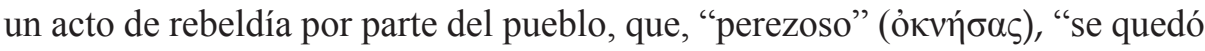

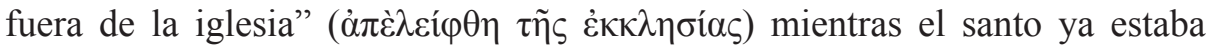

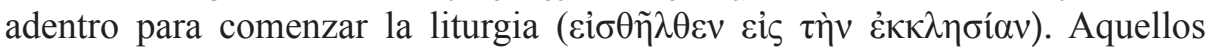
de quienes se espera que tengan afinidad con el santo, en tanto miembros y partícipes de la liturgia, muestran también una diferenciación ${ }^{28}$, con lo que se vuelve a poner en cuestión el concepto de alteridad: mientras que los definidos como pecadores llegan a acercarse a la figura del santo, quienes en principio se presumen cercanos a él se alejan por desobediencia.

Nos interesa también señalar un tipo de paralelismo dentro del mismo capítulo 12. Inmediatamente después de lo narrado sobre la pereza de la muchedumbre comienza la ceremonia y se produce una nueva contraposición entre los presentes y Espiridón: estos pretendían mantener la iluminación de una vela y Espiridón, en cambio, manda a encender todas las lucernas. Si bien no se trata de una oposición grave (es decir, no se trata de un pecado), ésta ayuda a intensificar la que fue referida previamente, recurso cohesivo al que Leoncio nos tiene acostumbrados ${ }^{29}$. Asimismo, pone de relieve una vez más el

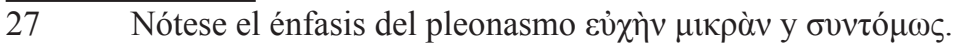

28 Se puede comparar esta actitud con la de los que rinden culto en el capítulo 13.

29 Sobre los procedimientos de cohesión en la Vida de Espiridón cf. Sapere (2015, 67 ss.). 
abismo que separa a Espiridón de los suyos.

Por último, en el capítulo 16 Espiridón conoce a Olimpo Paleuro,

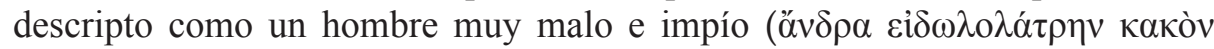

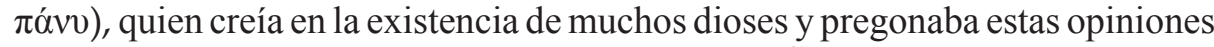

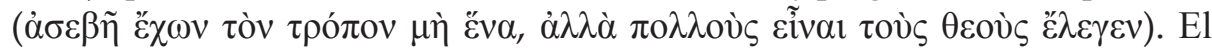
epíteto con el que es mencionado el personaje exacerba su impiedad, pues

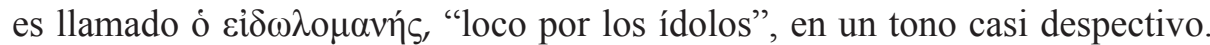
Curiosamente, no se hace referencia en la economía de la obra a ninguna mala acción de su parte, salvo su extrema impiedad. Su esposa, la fiel, honesta y

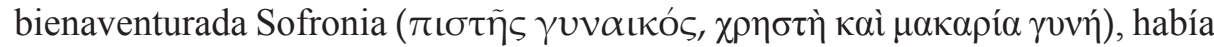
invitado a Espiridón a una comida en su casa, en la que también participaba Olimpo; en dicha ocasión se produce un milagro de clarividencia por parte del santo, lo que deja admirado al idólatra, quien a partir de semejante hecho comienza a respetarlo, pero sin abandonar la idolatría pagana (Oủ j̇ $\delta v \eta \dot{\theta \eta}$

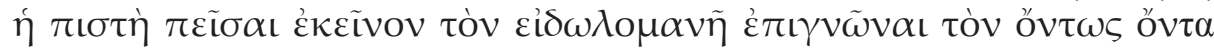

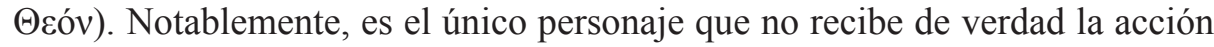
transformadora del santo, a la vez que el santo tampoco puede lograr una cercanía, como sí había ocurrido con otros personajes "opuestos". Se trata, a nuestro entender, de un claro ejemplo de otredad irresoluble, pues se presenta como un otro impío, malo e incapaz de convertirse ${ }^{30}$.

\section{Conclusiones: sobre la funcionalidad del paralelismo en la descripción del personaje de Espiridón}

A lo largo del trabajo hemos recorrido por completo los episodios de la Vida de Espiridón, análisis exhaustivo que nos permite extraer conclusiones sólidamente refrendadas desde el punto de vista textual. Dicho esto, expondremos las conclusiones a las que hemos arribado.

1) Se advierte una división estructural en la narración, ya que los episodios en los que el santo encuentra elementos de identificación con los distintos personajes aparecen en el comienzo de la biografía (capítulos 1 a 9) ${ }^{31}$ y los episodios en los que se diferencia de los personajes están agrupados hacia el final $(10 \text { a } 17)^{32}$, elaborando así un consistente entramado cohesivo.

$30 \quad$ Acerca de las categorías del 'otro' como 'pagano' y 'heleno' (al menos, nos permitimos conjeturar su origen griego a partir del nombre 'Olimpo') en Bizancio, cf. Kaldellis (2008, 120 ss.).

$31 \quad$ Exceptuando el 7.

32 Con excepción del 11. 
2) En aquellos casos en los que se produce un paralelismo por identificación (con los humildes campesinos, con Artemidoro, con las figuras de poder, etc.), aparece, no obstante, un elemento disrruptor, que pone en cuestión dicha identificación.

2.1) La única excepción a ello está representada por el episodio protagonizado por la hija del santo (cap. 1), en el que se destaca la pureza indiscutible de ambos como condición de posibilidad para la concreción del milagro.

3) En aquellos casos en los que el personaje del santo se enfrenta a una alteridad manifiesta (ante los pecadores y desobedientes), observamos un mecanismo discursivo que termina asimilando en cierta medida al santo con cada uno de los personajes, ya sea por tratarse de miembros de la comunidad eclesiástica (cap. 11 y 12) o porque esos mismos pecadores influyen positivamente en Espiridón (cap. 7 y 15) y hasta entablan un vínculo de cercanía (10 y 17), donde parece establecerse una especie de reconciliación de esos dos mundos disímiles.

3.1) Hay un único ejemplo en el que no se produce dicha reconciliación, que es la actitud inamovible del idólatra Olimpo (cap.16).

4) Los episodios destacados de Irene y Olimpo encuentran una ubicación estratégica: el de la hija del santo es el segundo capítulo de la hagiografía (es el capítulo 1 después de Prólogo); el de Olimpo, el anteúltimo. Conforman así una relación especular y de contraste (paralelismo).

De los puntos precedentes podemos colegir, pues, que no es casual el tratamiento de la figura del santo en contraste con los demás personajes de la obra. Por un lado, la presencia de personajes afines a Espiridón en el comienzo de la narración parece tener la intención de reforzar sus características positivas: pureza, sencillez, humildad, pero a la vez poder de acción. La identificación positiva apela también a captar la identificación del público, desde las clases más bajas hasta la elite, en tanto que los personajes que allí aparecen pertenecen a reconocibles tipos sociales. Los matices hallados a la hora de entablar las identificaciones apuntan, como adelantamos, a otorgarle al santo un status diferenciado: Espiridón es como todos y a su vez como ninguno. La segunda parte del relato contiene, entonces, los encuentros que conllevan un enfrentamiento con la alteridad, agrupados en ese lugar estratégicamente, para no generar desde el inicio una imagen polémica o combativa del santo; aparecen allí, no obstante, elementos de acercamiento con dichos personajes, 
pues encontramos aspectos de afinidad en quienes se diferencian del santo, llegando incluso a la empatía y a cierto vínculo de amistad. El santo es firme en su postura de oposición al pecador y al desobediente ${ }^{33}$, pero siempre puede haber un matiz en esa oposición. El único ejemplo en el que esto no ocurre es el episodio de Olimpo, pues se muestra abiertamente alejado de Dios. Irene, su contraejemplo, es signo de la pureza, modelo perfecto de santidad. En el medio de estos extremos ${ }^{34}$ (idolatría irreconciliable y pureza santa), los lectores podrán encontrar un personaje que los identifique a ellos y, a partir de la relación entablada con Espiridón, verse incluidos en el relato.

En suma, entendemos, que los paralelismos del texto, que representan vínculos de alteridad e identificación, son un elemento estructurante de la narración; la manera en la que están agrupados los episodios que representan unos y otros contribuye con el propósito didáctico, dado que la reiteración y la acumulación son estrategias retóricas clave de los textos hagiográficos.

\section{Referencias Bibliográficas}

BECK, H. (2002). “Interne Synkrisis bei Plutarch", Hermes, 130, 467-489.

BOURBOUHAKIS, E. AND I. NILSSON (2010). "Byzantine Narrative: the Form of Storytelling in Byzantium”, en James, L. (ed.). A Companion to Byzantium. London: Blackwell Publishing, 263-274.

CAVALLERO, P. ET AL (2014). Leoncio de Neáplis: Vida de Espiridón. Edición crítica con traducción, introducción, notas y apéndices. Buenos Aires: Editorial de la Facultad de Filosofía y Letras de la Universidad de Buenos Aires.

DUFF, T. (2000). "Plutarchean Synkrisis: comparisons and contradictions", en Van der Stockt, L. (ed.) (2000). Rhetorical theory and praxis in Plutarch. Acta of the IVth International Congress of the International Plutarch Society, Leuven, July 3-6, 1996. Leuven: Peeters Publishers, 141-162.

ERBSE, H. (1956). "Die Bedeutung der Synkrisis in den Parallelbiographien Plutarchs", Hermes, 84, 398-424.

GARITTE (1955). "L'édition des Vies de saint Spyridon par M. van den Ven", Revue d'histoire ecclésiastique, 50, 125-140.

GORMAN, H. M. (2015). Interweaving Innocence: A Rhetorical Analysis of Luke's Passion Narrative (Luke 22:66--23:49). Eugene: Wipf and Stock Publishers.

33 En efecto, hemos visto que señala los pecados y amonesta a los pecadores.

34 Destacados por la posición especular en la que se hallan, como vimos. 
HÄGG, T. (2011): "The Life of St. Anthony between Biography and Hagiography", en Efthymiadis, S. (2011). The Ashgate Research Companion to Byzantine Hagiography. Burlington, London: Ashgate Publishing, 17-34.

HÄGG, T. (2012). The Art of Biography in Antiquity. Cambridge: University Press.

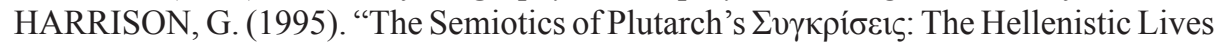
of Demetrius-Antony and Agesiaus-Pompey", Revue belge de philologie et d'histoire, 73. 1, 91-104.

HUNGER, H. (1978). Die hochsprachliche profane Literatur der Byzantiner, 1 Band: Handbuch der Altertumwissenschaft XII. München: Beck.

KALDELLIS, A. (2008). Hellenism in Byzantium. The Transformations of Greek Identity and the Reception of the Classical Tradition. Greek Culture in the Roman World. New York: Cambridge University Press.

KAZHDAN, A. (1990). "Byzantine Hagiography and Sex in the Fifth to Twelfth Centuries", Dumbarton Oaks Papers, 44, 131-143.

KENNEDY, G. A. (1999). Classical Rhetoric and Its Christian and Secular Tradition from Ancient to Modern Times. Chapel Hill: University of North Carolina Press.

KENNEDY, G. A. (2003). Progymnasmata: Greek Textbooks of Prose Composition and Rhetoric. Leiden: Brill.

KUSTAS, G. L. (2001). "The function and evolution of Byzantine rhetoric”, en Nagy, G. (2001). Greek Literature in the Byzantine Period. London: Routledge, 179198.

LARMOUR, D. (1992). "Making Parallels: Synkrisis and Plutarch's 'Themistocles and Camillus"”, $A N R W$, II.33.6, 4154-4200.

LONGO, A. (2004). "Vite, passioni, miracoli dei santi”, en Cavallo, G. (ed.). La cultura Bizantina, vol. I de Lo spazio letterario del medioevo, 3: Le culture circostanti. Roma: Salerno, 183-227.

NEYREY, J. H. (2009). The Gospel of John in Cultural and Rhetorical Perspective. Michigan: Wm. B. Eerdmans Publishing.

PATLAGEAN, E. (1968). "À Byzance: ancienne hagiographie et histoire sociale”, Annales. Économies, Sociétés, Civilisations, 23e année, $\mathrm{n}^{\circ}$ 1, 106-126.

PELLING, C. (1986), "Synkrisis in Plutarch's Lives", en Brenk, F. and I. Gallo (eds.).Miscellanea Plutarchea: Atti del I convegno di studi su Plutarco (Roma, 23 novembre 1985), Quaderni del Giornale Filologico Ferrarese. Ferrara, Giornale filologico ferrarese, 83-96.

PRATSCH, T. (2005). Der hagiographische Topos: Griechische Heiligenviten in mittelbyzantinischer Zeit, Millennium Studies. Berlin: Walter de Gruyter.

RAPP, C. (1999). “"For next to God, you are my salvation': reflections on the rise of the Holy man in Late Antiquity”, en Howard-Johnston, J. \& P. A. Hayward (eds.). The Cult of Saints in Late Antiquity and the Middle Ages. Oxford: University Press, 65-84. 
RAPP, C. (2016). Brother-Making in Late Antiquity and Byzantium: Monks, Laymen, and Christian Ritual. Oxford: University Press.

ROUSSEAU, P. (1999). "Ascetics as mediators and as teachers", en Howard-Johnston, J. \& P. A. Hayward (eds.). The Cult of Saints in Late Antiquity and the Middle Ages. Oxford: University Press, 65-84.

RUBENSON, S. (2000). "Philosophy and simplicity: the problem of classical education in early Christian biography", en T. Hagg and P. Rousseau (eds.) (2000). Greek Biography and Panegyric in Late Antiquity. Berkeley, Los Angeles, London: University of California Press.

SAPERE, A. Y VILLAHOZ, J. (2014). "Relación autor-personaje en tres Vidas de Leoncio de Neápolis" (en colaboración), en Assis, M. E. y C. E. Lobo. (comp.) (2014), Significación y resignificación del mundo clásico antiguo. Actas XXII Simposio Nacional de Estudios Clásicos, vol. 2. San Miguel de Tucumán: Editorial de la Facultad de Filosofía y Letras de la UNT, 947-954.

SAPERE, A. (2015). "Koinóteros logos: un análisis retórico y estilístico de la Vida de Espiridón", Revista Helmantica, LXVI, 61-91.

SHIPP, B. (2005). Paul the Reluctant Witness: Power and Weakness in Luke's Portrayal. Eugene: Wipf and Stock Publishers.

SWAIN, S. (1992). "Plutarchan Sunkrisis", Eranos, 90, 101-11.

VAN DEN VEN, P. (1953). La légende de S. Spyridon, évêque de Trimithonte. Louvain. Institut orientaliste.

WHITBY, M. (2010). "Rhetorical Questions", en James, L. (ed.). A Companion to Byzantium. London: Blackwell Publishing, 239-250.

YOUNI, M. (2001). "Adultery in Byzantine Law: Development and Survivals", en Papastathis, C. (ed.). Proceedings of the International Symposium of Jurists, Thessaloniki, 10-13 de diciembre de 1998, 279-298. 